\title{
Technè
}

La science au service de l'histoire de l'art et de la préservation des biens culturels

\section{Concilier dépollution et restauration du patrimoine industriel : le cas du chantier de restauration du musée Stella Matutina (île de La Réunion)}

Reconciling the depollution and restoration of industrial heritage: the case of the restoration project at the Musée Stella Matutina (île de La Réunion)

\section{Agnès Jean-Jacques, Aurélie Mac-Luckie et Arnauld Martin}

\section{OpenEdition}

Journals

Édition électronique

URL : http://journals.openedition.org/techne/465

DOI : $10.4000 /$ techne.465

ISSN : 2534-5168

Éditeur

C2RMF

Édition imprimée

Date de publication : 1 décembre 2018

Pagination : $96-103$

ISBN : 978-2-11-152829-1

ISSN : $1254-7867$

\section{Référence électronique}

Agnès Jean-Jacques, Aurélie Mac-Luckie et Arnauld Martin, « Concilier dépollution et restauration du patrimoine industriel : le cas du chantier de restauration du musée Stella Matutina (île de La

Réunion) », Technè [En ligne], 46 | 2018, mis en ligne le 19 décembre 2019, consulté le 23 juillet 2020.

URL : http://journals.openedition.org/techne/465; DOI : https://doi.org/10.4000/techne.465

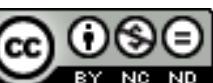

La revue Technè. La science au service de l'histoire de l'art et de la préservation des biens culturels est mise à disposition selon les termes de la Licence Creative Commons Attribution - Pas d'Utilisation Commerciale - Pas de Modification 4.0 International. 


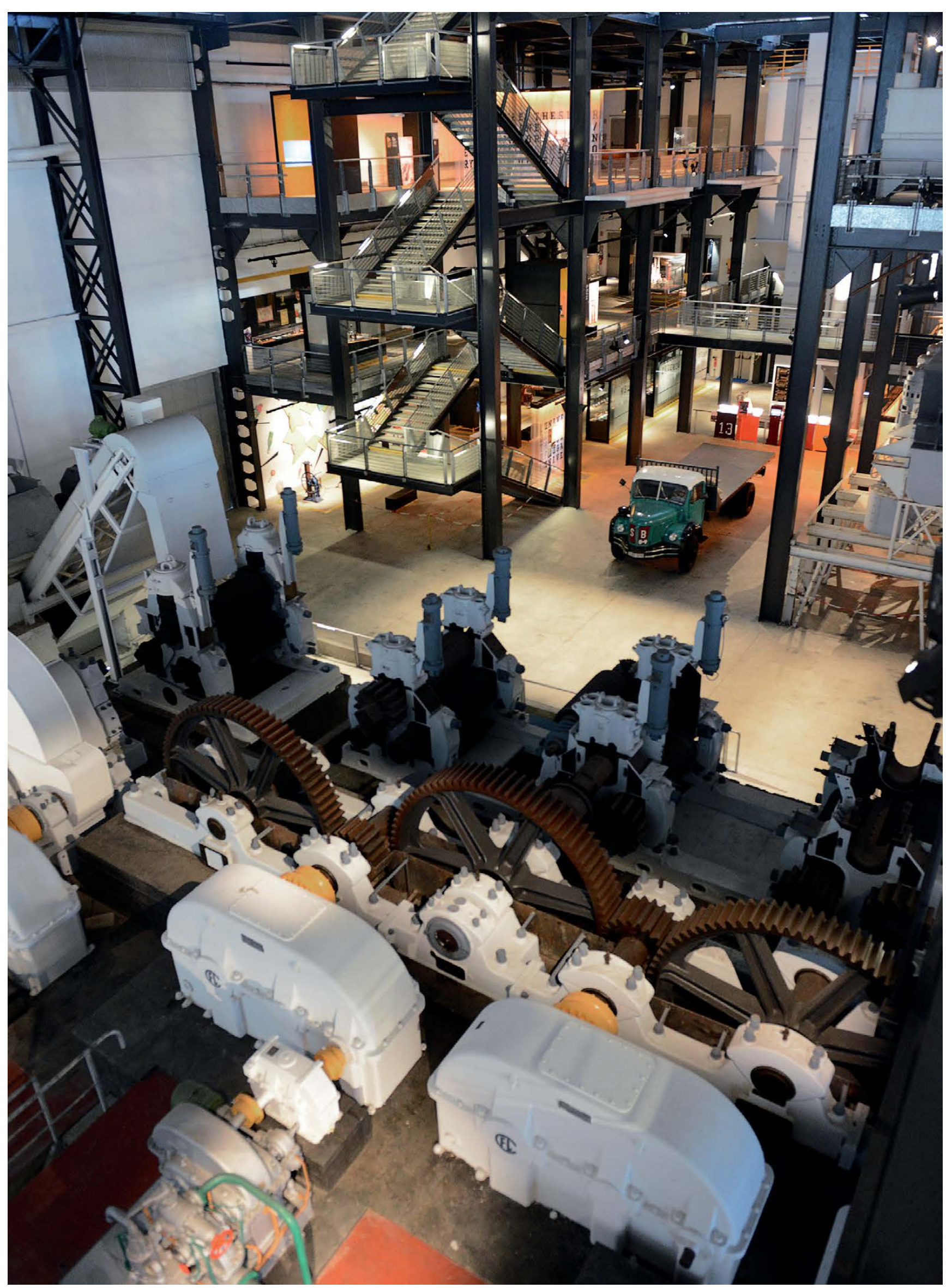

Fig. 1. Intérieur du nouveau musée. ( G. Ancel. 
Agnès Jean-Jacques

Aurélie Mac-Luckie Arnauld Martin

\section{Concilier dépollution et restauration du patrimoine industriel : le cas du chantier de restauration du musée Stella Matutina (île de La Réunion)}

Reconciling the depollution and restoration of industrial heritage: the case of the restoration project at the Musée Stella Matutina (île de La Réunion)

Résumé. Première opération de cette ampleur à La Réunion, le chantier des collections du musée Stella Matutina mené durant sa réhabilitation architecturale était complexe à plus d'un titre. Aux contraintes de climat tropical et d'éloignement géographique s'ajoutait la difficulté à concilier le respect des normes patrimoniales, la dépollution de l'amiante et du plomb et le traitement des machines intégrées à l'architecture. Sur place se trouvent des entreprises qualifiées dans la maintenance industrielle, mais peu de restaurateurs du patrimoine. Face à ces difficultés, la réflexion conduite a abouti à des associations innovantes entre entreprises locales spécialisées et restaurateurs qualifiés. Le montage administratif optimisé et l'implication de tous les intervenants ont permis de définir des protocoles inédits pour le traitement de l'amiante sur des objets patrimoniaux.

Mots-clés. Patrimoine industriel, musée technique, restauration, dépollution, amiante, plomb, marchés publics, île de La Réunion.
Abstract. The first operation of this scale to be carried out in La Réunion, the restoration of the Musée Stella Matutina collections undertaken during its architectural rehabilitation, was complex on more than one level. In addition to the constraints related to the tropical climate and geographical remoteness was the difficulty in reconciling respect for heritage norms, the removal of asbestos and lead and the handling of the machines integrated into the architecture. Industrial maintenance companies could be found on site, but few national heritage restorers. The think tank set up to solve these problems resulted in innovative collaborations between specialised local companies and skilled restorers. The optimised administrative set-up of the project and the involvement of all the participants enabled us to define unprecedented protocols for the handling of asbestos in objects belonging to national heritage.

Keywords. Industrial heritage, technology museum, restoration, remediation, asbestos, lead, public procurement, La Réunion.
De 2010 à 2015, la Région Réunion a entièrement réhabilité le musée Stella Matutina ${ }^{1}$, sur les plans architecturaux et muséographiques, replaçant l'usine au cœur du propos scientifique.

Installé dans une sucrerie fermée en 1978, le musée conserve 1500 objets représentatifs du patrimoine sucrier de La Réunion : machines industrielles intégrées au bâtiment, véhicules et équipements agricoles, outils et objets du quotidien.

En quatre ans, plus de 600 objets ont été restaurés pour être exposés dans la nouvelle muséographie, dont 42 machines et pièces industrielles lourdes. La restauration de ces dernières donne à ce chantier un caractère exceptionnel. Il a en effet fallu innover et mettre en œuvre des procédures inédites pour faire face à des contraintes climatiques, d'éloignement, de pollution - amiante et plomb - et d'interaction avec le chantier de réhabilitation du site.

\section{Un territoire aux contraintes fortes}

\section{Des contraintes climatiques...}

L'île de La Réunion (2 512 km²) est située dans l'océan Indien, proche du tropique du Capricorne. Le climat est tropical humide et le relief accidenté influe sur le régime des vents et des pluies.

Le musée se trouve dans une zone faiblement ventée. Un effet de foehn se produit par subsidence et crée un contrevent. L'air qui redescend des montagnes est alors plus sec et la pluviométrie bien moindre (440 mm max/jour) que sur la côte opposée. La température moyenne annuelle est de $25,4^{\circ} \mathrm{C}$ et le taux d'humidité relative moyen reste élevé (65\%). À ce facteur de dégradation des œuvres et notamment du métal s'ajoute l'air salin, généré par la barrière de corail située non loin du musée. Le bâtiment principal du

Agnès Jean-Jacques, attachée de conservation du patrimoine, Région Réunion, Direction de la Culture et du Patrimoine culturel Pôle musées (agnes.jean-jacques@cr-reunion.fr). Aurélie Mac-Luckie, diplômée de l'ESBA Talm en conservation-restauration des œuvres sculptées, restauratrice du patrimoine indépendante spécialisée en climat tropical et biens patrimoniaux en volume (am.restauration@ gmail.com). Arnauld Martin, attaché de conservation du patrimoine, responsable scientifique, lors du chantier, du musée Stella Matutina (arnauld.martin@gmail.com). 


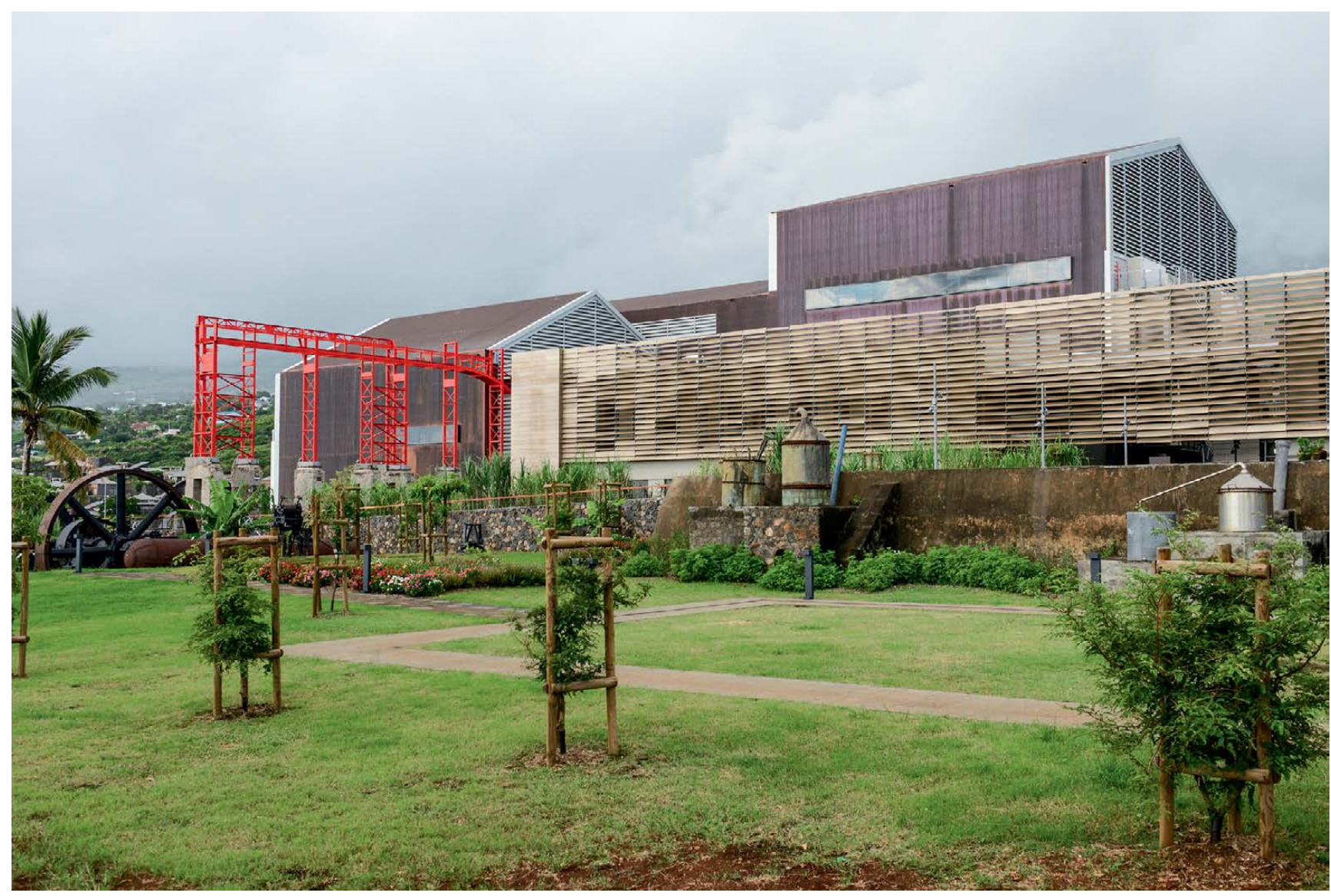

Fig. 2. Vue extérieure du nouveau musée. @ G. Ancel.

musée étant conçu pour une ventilation naturelle, les objets qui y sont exposés sont soumis quotidiennement aux effets de ces polluants extérieurs.

Ces contraintes climatiques ont conditionné le choix des produits de protection : polymères testés et sélectionnés pour leur bonne tenue à l'humidité (cires synthétiques microcristalline et polyester pour les objets en intérieur, résine acrylique en solution, résine de type alkyde en dispersion solvantée, silanes organofonctionnels, caoutchouc chloré ou tout autre produit présentant les caractéristiques citées), ou systèmes de mise en peinture décrits par des normes et des certifications (par exemple ACQPA, environnement « marine» de corrosivité très élevée).

Dans le cas des objets métalliques ne pouvant être démontés pour être protégés dans leur intégralité, il est envisagé que les zones présentant des risques de corrosion caverneuse ou d'usure rapide de la protection (comme des surfaces soumises à des frottements) soient graissées, cette méthode ayant donné de bons résultats sur le long terme par le passé2. ...et des contraintes d'isolement

La Réunion compte cinq « Musées de France ». Mais la spécificité de leurs collections, l'éloignement géographique (à 9300 km de Paris) et l'étroitesse du marché de la restauration n'attirent pas durablement les restaurateurs diplômés. Pour les mêmes raisons, les fournisseurs de produits de restauration sont absents du territoire.

Les marchés de restauration sont habituellement attribués à des professionnels externes : soit les œuvres sont envoyées en France métropolitaine, soit le restaurateur vient avec son matériel et ses produits. La première solution comporte des risques liés au transport (chocs thermiques, manipulations) et implique des objets de taille réduite, la seconde présente d'autres difficultés : déclaration en douane de certains produits ${ }^{3}$ et anticipation des besoins sur place sans forcément avoir vu les œuvres. En outre, des traitements peuvent alors être réalisés sans tenir compte des conditions de température et d'humidité « hors normes », ce qui limite considérablement leur durée de vie. 
Toutes ces raisons, auxquelles s'ajoute l'impossibilité évidente de déplacer des machines de plusieurs tonnes à des milliers de kilomètres de leur lieu de conservation, ont amené l'équipe projet à travailler a maxima avec des compétences et produits locaux. Tout l'enjeu consista alors à concilier un savoir-faire éprouvé aux conditions réunionnaises avec les normes de qualité et la déontologie de la conservation-restauration définies par le code $\mathrm{ECCO}^{4}$ et la charte Nizhny Tagil pour le patrimoine industriel de juillet $2003^{5}$.

\section{Les machines de l'usine}

\section{Un ensemble cohérent...}

Stella Matutina est un témoin de l'industrie sucrière des années 1960-1970. À la fermeture en 1978, peu de machines ont été démontées, alors que la revente ou le ferraillage de l'équipement étaient pratiques courantes. Toutes les étapes de la fabrication du sucre étant encore visibles dans l'usine, il a été décidé en 1985 d'en faire un musée.

L'équipement industriel est lourd, solidement ancré dans le sol et lié à la structure du bâtiment. Toute tentative de déplacer une machine afin de la traiter en atelier était inenvisageable pour la collectivité dans l'enveloppe et le calendrier impartis. De plus, de telles opérations auraient pu fragiliser la structure et causer des incidents ou des déformations lors de la manutention.

\section{... mais pollué}

Les machines concernées ont fonctionné quotidiennement dans l'usine pendant des années. Le personnel des ateliers réalisait un entretien annuel pour protéger les métaux de la corrosion, appliquant un apprêt antirouille à base de minium de plomb et repeignant régulièrement les machines ${ }^{6}$.

Bon nombre d'entre elles fonctionnaient à la vapeur ou intégraient une circulation de fluides chauds. Utilisé jusque dans les années 1980 pour ses qualités ignifuges, calorifuges, sa flexibilité et sa résistance à la tension, l'amiante était donc très souvent employé pour les joints de bride, les systèmes de freinage et les peintures de revêtement.

Dans le bâtiment, le retrait des polluants s'effectue dans un cadre normé, par des professionnels certifiés, formés et expérimentés sur des protocoles n'incluant pas de notion de patrimoine, de sauvegarde et d'intervention non destructrice.

\section{Une stratégie à penser}

\section{Avec la maîtrise d'œuvre}

La rédaction du nouveau projet scientifique et culturel débute en août 2010 suite à la décision de réhabiliter le musée. Ce document et le programme muséographique qui en découle s'appuient sur les réflexions d'un conseil scientifique pointant la nécessité de donner du sens à ce musée souffrant d'une image confuse et datée. Ils mettent notamment l'accent sur la vocation industrielle du site.

L'ancien musée utilisait l'usine et ses machines comme décor. La fabrication du sucre était devenue incompréhensible. La remise en ordre des étapes du procédé de fabrication et leur lisibilité accrue sont donc inscrites au cœur du projet, avec des conséquences en termes de muséographie, de scénographie et de restauration.

Parallèlement, l'équipe de maîtrise d'œuvre, incluant un muséographe, adopte un principe majeur dans le projet architectural et muséographique : faire de l'usine le principal objet du musée. Cette convergence n'est pas fortuite : la compréhension et le respect du PSC dans le projet architectural constituent une attente importante de la maîtrise d'ouvrage. Rendre explicites les machines comme objets techniques et faire réapparaître dans l'usine la dimension de «cathédrale » de l'industrie occultée par la précédente architecture sont donc des objectifs entièrement partagés (fig. 1 et 2).

D'un point de vue architectural, cela impliquait de démonter les paliers et cloisons qui s'étaient intercalés entre les unités organiques de l'usine, pour les rendre visibles et compréhensibles.

Remplaçant les anciens cheminements qui, parfois, masquaient totalement des étapes importantes du processus, un système de passerelles métalliques légères surplombant les machines est mis en place. Il permet aux visiteurs de suivre, étape par étape, la fabrication du sucre de l'entrée de la canne dans l'usine à la sortie du sucre et des bas-produits. Des dispositifs, statiques et multimédia - appelés « observatoires »favorisant le repérage et l'identification visuelle des machines dans l'espace de l'usine donnent les clés de compréhension de leur fonctionnement et permettent d'enrichir le propos en diffusant les témoignages des anciens travailleurs (fig. 3).

Le principe du traitement simultané de l'architecture et des machines est ainsi acté, reste à le mettre en ouvre.

\section{Avec le C2RMF}

Sollicité par la Région Réunion, le C2RMF diligente une mission d'expertise des collections industrielles du musée, en vue de leur restauration. Cette intervention rapide permet de soulever toutes les questions évoquées : dépollution, contraintes climatiques et logistiques, absence de restaurateurs spécialisés, coûts ${ }^{7}$. Philippe Goergen, chef du département de la Conservation préventive au C2RMF, rencontre sur place la maîtrise d'ouvrage, la maîtrise d'œuvre, le diagnostiqueur amiante et les entreprises de maintenance industrielle susceptibles d'intervenir sur les machines. Après évaluation des projets de cahiers des charges du maître d'œuvre, il préconise la rédaction des constats d'état par un assistant à maîtrise d'ouvrage en restauration. D'autre part, une étude des restaurations effectuées depuis 1991 et des compétences locales disponibles est menée. 


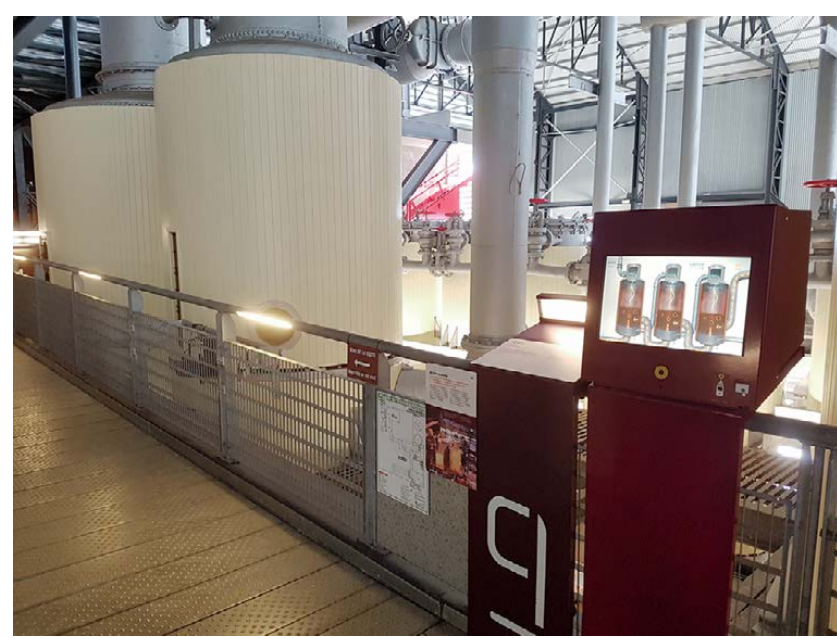

Fig. 3. Vue de l'« observatoire " des évaporateurs (dispositif multimédia). (c) A. Jean-Jacques.

S'appuyant sur cette analyse et sur des principes méthodologiques décrits dans un article paru en $2011^{8}$, le C2RMF propose que les entreprises locales expérimentées en restauration des machines sucrières puissent candidater, sous réserve d'une supervision des travaux par un restaurateur habilité. Ce dernier établirait les cahiers des charges détaillés des interventions, assurerait le suivi du chantier, et proposerait les protocoles de conservation préventive réalisables par l'équipe du musée.

Cette mission souligne enfin l'absence de grille méthodologique concernant le traitement de l'amiante dans les collections publiques.

\section{Avec le soutien de la collectivité}

Pour donner corps à ces principes en optimisant les délais des procédures administratives, l'accord-cadre ${ }^{9}$ qui permet davantage de souplesse dans la passation des marchés ultérieurs relatifs aux différents lots de travaux, est choisi. Il incombe dès lors au maître d'œuvre de prendre en charge les marchés subséquents de dépollution et de restauration. Son implication dans le chantier des collections et son adhésion au projet muséographique sont donc essentielles.

Par ailleurs, toutes ces machines étant inventoriées, il est nécessaire d'inventer une méthodologie conciliant dépollution et restauration. La collectivité décide de s'entourer de deux assistants à maîtrise d'ouvrage : un bureau d'étude spécialisé dans la dépollution et une restauratrice pour mettre en place et contrôler le déroulement des restaurations, dont les protocoles sont soumis en amont à la Commission scientifique régionale des collections des Musées de France, qui émet un avis favorable ${ }^{10}$.

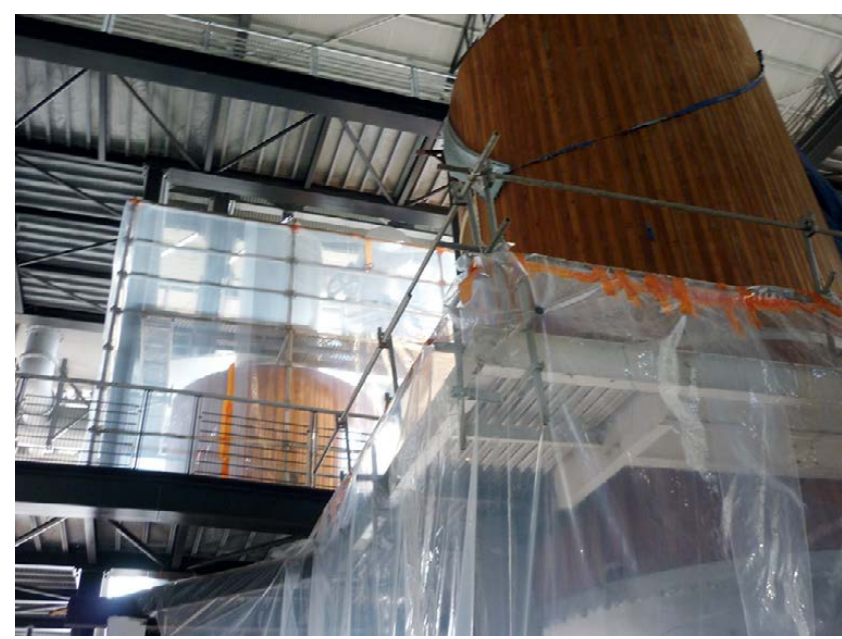

Fig. 4. Confinement d'une machine amiantée (évaporateurs). (C) A. Mac-Luckie.

\section{Mettre en œuvre le chantier}

Les assistants étudient d'abord l'existant : les machines potentiellement amiantées et plombées subissent des prélèvements et des analyses pour identifier les éléments pollués. Les deux experts réalisent et confrontent cartographies et listings. Les constats d'état sont réalisés en parallèle. Observations visuelles, stratigraphies et recherches historiques sur les couleurs documentent les protocoles de restauration.

Ce diagnostic est suivi d'un partage des informations et d'un échange sur les méthodologies de dépollution et de conservation-restauration, pour s'accorder sur les interventions les moins nuisibles aux collections : désamiantage par encapsulage plutôt que retrait des zones amiantées pour éviter la perte définitive d'éléments (par exemple, tuyaux et brides en acier $)^{11}$. Un film étanche est appliqué sur la fibre d'amiante en plusieurs épaisseurs (fig. 5). Les produits sont sélectionnés pour pouvoir être peints ou permettre des interventions ultérieures. Pour le déplombage, un nettoyage à l'eau plutôt qu'à sec est mis en place avec un contrôle systématique des eaux de récupération et des surfaces (lingettes récupérant les poussières) jusqu'à obtenir un taux de plomb inférieur à $1 \mathrm{mg} / \mathrm{cm}^{2}$.

Le traitement de l'amiante est spécifique et nécessite des certifications. Une entreprise indépendante des travaux de restauration est donc choisie pour cette opération. En revanche, le déplombage étant indissociable du nettoyage et $\mathrm{du}$ traitement de la corrosion, c'est l'entreprise responsable de la restauration des machines qui en a la charge. L'installation des confinements de sécurité est mutualisée afin de limiter les coûts. Le plomb est traité en premier, puis le confinement déjà en place est contrôlé et renforcé par la seconde entreprise pour l'amiante (fig. 4). Chaque entreprise assure ensuite l'évacuation de ses déchets pollués dans des filières dédiées. 


\section{Exemple : cas du traitement des évaporateurs}

I

es évaporateurs sont situés au cœur du musée. Il s'agit d'un ensemble constitué de cinq machines

$\backslash$ en alliage ferreux recouvertes de lambris de bois et reliées entre elles par un système de tuyauterie aujourd'hui incomplet.

La méthodologie de dépollution et de restauration mise en œuvre a fait intervenir plusieurs opérateurs présentant des niveaux de certifications spécifiques aux polluants à traiter :

1. Le repérage des pollutions, leur localisation précise et les analyses ont été réalisés par une entreprise de contrôle technique et diagnostics de bâtiment, après adaptation du protocole appliqué pour les immeubles et ouvrages d'art.

2. Le protocole de traitement a été mis en place par les deux AMO restauration et dépollution pour traiter les fibres d'amiante de type Chrysotile repérées dans les joints via :

- un minimum de démontage ;

- une utilisation de résines d'encapsulage recouvrables par les peintures employées au cours de la restauration;

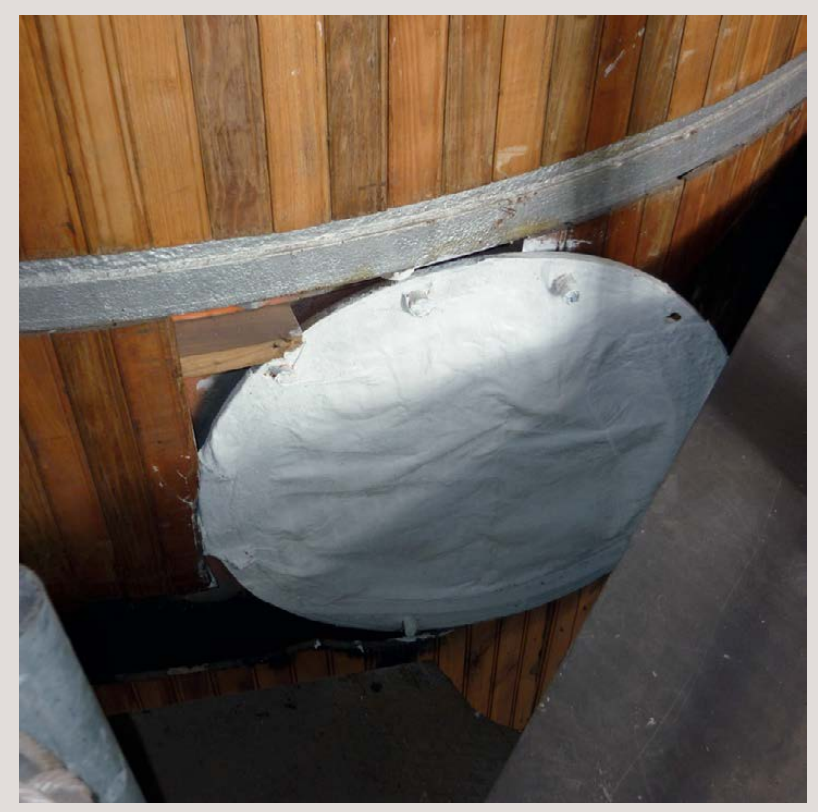

Fig. 5. Détail d'un encapsulage sur un évaporateur () A. Mac-Luckie.
- un recours au retrait uniquement lorsque le joint est accessible et la pièce concernée démontable sans dommages pour les matériaux à proximité ;

- un repérage par étiquetage des zones potentiellement amiantées ou n'ayant pas fait l'objet d'un retrait.

3. Une surface de confinement est délimitée autour des évaporateurs, puis le traitement de l'amiante est réalisé par une société de désamiantage. Pour les joints de bride des tuyaux, l'amiante est encapsulée avec un silicone blanc dit « recouvrable » sur une épaisseur de plusieurs millimètres. La surface est lissée et les débordements nettoyés pour limiter au maximum l'impact visuel. Après une mise en peinture, le joint n'est plus visible.

Pour les cas particuliers, la méthode est adaptée : sur un conduit de fluide (air) de diamètre important, l'entreprise, en concertation avec les AMO, a choisi d'obturer l'ouverture avec une plaque de fibre de verre enduite de résine polyester non saturée thixotropique fabriquée sur mesure. Celle-ci est boulonnée sur le conduit en réutilisant les trous de fixation d'origine, les joints d'étanchéité sont réalisés au silicone, puis la plaque est recouverte d'une tôle en alliage ferreux et mise en peinture comme le reste des évaporateurs après l'application d'un dernier joint de silicone sur la périphérie du système (fig. 5).

Seuls ces deux polymères ont été utilisés pour l'encapsulage afin de ne pas multiplier le nombre de produits de restauration. Ils ont été sélectionnés par les AMO parmi la liste des produits couramment employés par la société car ils présentaient de bons résultats en termes d'étanchéité pour les encapsulages et n'entravaient pas la restauration des évaporateurs après traitement amiante.

4. Après intervention, le confinement et les matériaux employés sont éliminés dans des filières dédiées et les autres restaurations (dépollution plomb par aspiration THE, traitement des corrosions et protection des surfaces) peuvent être entreprises. 
Les interactions entre les intervenants étant importantes, des plannings précis et détaillés et un suivi journalier par les assistants sont essentiels au bon déroulement des opérations.

\section{S'appuyer sur les savoir-faire locaux}

L'une des entreprises locales d'ingénierie et rénovation industrielle, expérimentée en traitement des machines, est retenue pour l'opération et collabore étroitement avec l'assistant en restauration, suivant l'avis du C2RMF.

Dans un premier temps, le binôme définit les protocoles et réalise des tests sur des échantillons pour choisir les traitements de passivation des métaux les plus performants et l'aspect visuel le plus satisfaisant. L'assistant forme deux ouvriers à la préparation et à l'emploi de l'acide tannique et de la cire microcristalline à la place des habituels vernis alkydes au cobalt et revêtements à base de caoutchouc chloré ${ }^{12}$ (fig. 6).

Les opérations de restauration sont réalisées dans la continuité de la dépollution. Les deux assistants suivent les travaux au jour le jour, répondant aux questions et cherchant à résoudre les problèmes techniques et éthiques. Les deux entreprises intervenant sur une même pièce veillent également à ce que les opérations réalisées par l'une ne perturbent pas celles à effectuer par l'autre, notamment par le choix de résines d'encapsulage pouvant recevoir les mêmes couches de protection que le reste de la machine.

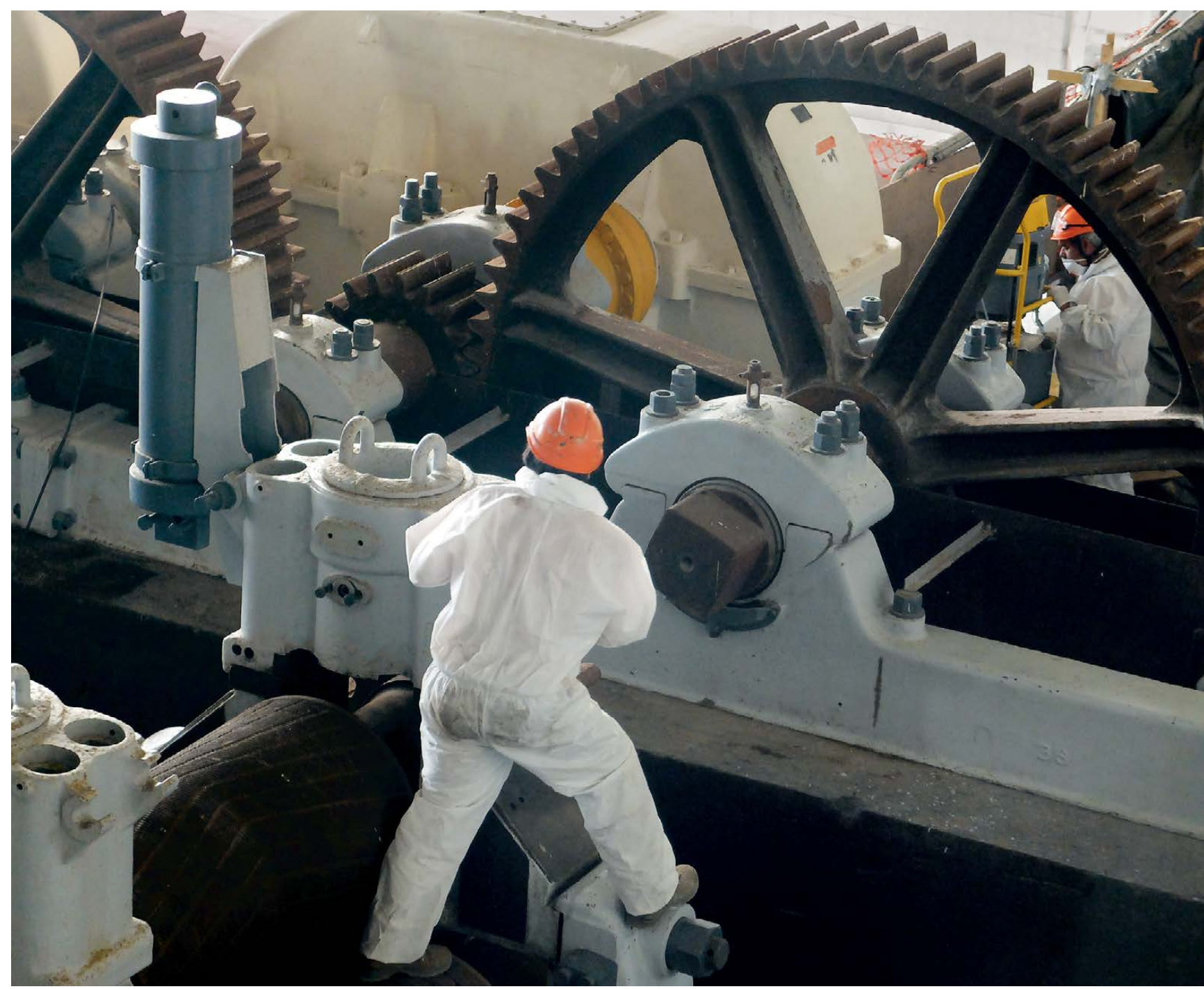

Fig. 6. Restauration d'un moulin. (c) G. Ancel. 


\section{Conclusion}

Inédit à La Réunion, ce chantier complexe a poussé à s'adapter et à chercher des solutions à des problèmes variés en s'appuyant sur l'existant, dans un cadre contraint en temps et en budget. Mais autour de cette réhabilitation, ce sont surtout des collaborations durables qui se sont mises en place entre les entreprises locales et les rares restaurateurs locaux, structurant une filière essentielle pour les musées réunionnais, et des protocoles efficaces qui ont été imaginés pour répondre à des problématiques récurrentes dans le patrimoine industriel ${ }^{13}$.

\section{Remerciements}

Les auteurs remercient le C2RMF, notamment Philippe Goergen, et la Direction des Affaires Culturelles de l'océan Indien pour leur appui tout au long de ce chantier des collections, ainsi que le comité de rédaction de Technè, pour leur contribution à cet article.
Notes

1. Stella Matutina ( étoile du matin » en latin) est la planète Vénus, qui apparaît à l'est dans le ciel matinal. C'est l'objet céleste le plus brillant après le Soleil et la Lune : elle annonce le lever du jour et le début de la journée de travail. Stella Matutina est associée à sainte Marie, sous la protection de laquelle l'usine avait été placée par ses dirigeants.

2. Procédé testé depuis les années 1990 et appliqué par le personnel du musée.

3. Les départements d'outre-mer ont une fiscalité différente de celle qui s'applique en métropole et dans l'Union européenne. Les produits arrivant sur le territoire réunionnais doivent être déclarés, et sont soumis à une imposition spécifique (loi n ${ }^{\circ}$ 2004-639 du 2 juillet 2004 relative à l'octroi de mer modifiée par la loi $n^{\circ} 2015$ 762 du 29 juin 2015).

4. Règles professionnelle de la Confédération européenne des organisations de conservateursrestaurateurs.

5. Charte présentée à l'Icomos en juillet 2003 et publiée par l'Unesco.

6. Des recherches menées auprès des entreprises locales ayant travaillé sur le premier musée, croisées avec des observations stratigraphiques, ont permis de constater qu'une sous-couche à base de minium était systématiquement employée dans le protocole de rénovation des machines au début des années 1990.

7. Rapport de Philippe Goergen,

"Mission Stella Matutina, île de La Réunion

- du 26 février au 4 mars 2012 », C2RMF.
8. Mirambet-Paris, Mirambet, 2011.

9. « Les accords-cadres sont les contrats conclus par un ou plusieurs acheteurs [...] avec un ou plusieurs opérateurs économiques ayant pour objet d'établir les règles relatives aux bons de commande à émettre ou les termes régissant les marchés subséquents à passer au cours d'une période donnée, notamment en ce qui concerne les prix et, le cas échéant, les quantités envisagées. » Ordonnance n ${ }^{\circ}$ 2015-899 du 23 juillet 2015 relative aux marchés publics.

10. Compte-rendu du 25 mai 2012 de la Commission scientifique régionale des collections des Musées de France - île de La Réunion - Direction des Affaires culturelles de l'océan Indien.

11. Le retrait n’a été réalisé que lorsque la pièce était démontable et pouvait être remise à son emplacement initial après traitement.

12. Cette méthode est couramment employée par les laboratoires de restauration traitant des objets archéologiques. Elle a été choisie ici pour traiter des objets qui avaient été partiellement enfouis et présentaient le même type de dégradations.

13. Afin de raconter ce chantier hors norme et rendre hommage à tous ceux qui y ont pris part, la première exposition temporaire du musée a été consacrée à la réhabilitation. Présentée de juin à octobre 2015, «Métamorphose(s). 2010-2015 Le making-of du nouveau musée Stella Matutina, une exposition dessinée par Hippolyte » narrait, en alternant bande dessinée et témoignages, toutes les étapes de cette opération unique à La Réunion.
Bibliographie

Exp. Région Réunion, 2015, Métamorphose(s) 2010-2015 - Le making-of du nouveau musée Stella Matutina, une exposition dessinée par Hippolyte [Cat. Exp., Région Réunion/musée Stella Matutina].

Ministère de la Culture et de la Communication, Conseil des métiers d'art, Commission patrimoine, «Document relatif à l'application du Code des marchés publics aux marchés de conservation-restauration de biens culturels ", version avril 2009.

Mirambet-Paris A., Mirambet F., 2011, «La conservation-restauration du patrimoine technique et industriel dans le cadre de la loi sur les Musées de France, une mission impossible? ", La Lettre de l'OCIM, n ${ }^{\circ} 135$, p. 27-35.

Tomsin P., 2007, « Les patrimoines mobiliers scientifique et technique : spécificités de leur restauration, de leur conservation et de leur valorisation ", CeROArt [En ligne] http://ceroart. revues.org/370 